\title{
MicroRNA-203 inhibits cellular proliferation and invasion by targeting Bmi1 in non-small cell lung cancer
}

\author{
TENGFEI CHEN ${ }^{1,2^{*}}$, CHUN XU $^{1,2^{*}}$, JUN CHEN $^{1,2}$, CHENG DING $^{1,2}$, \\ ZHENLEI XU ${ }^{1,2}$, CHANG LI ${ }^{1,2}$ and JUN ZHAO ${ }^{1,2}$ \\ ${ }^{1}$ Department of Thoracic and Cardiovascular Surgery, The First Affiliated Hospital of Soochow University, \\ Medical College of Soochow University, Suzhou, Jiangsu 215006; \\ ${ }^{2}$ Suzhou Key Laboratory for Molecular Cancer Genetics, Suzhou, Jiangsu 215123, P.R. China
}

Received July 26, 2014; Accepted March 3, 2015

DOI: $10.3892 / \mathrm{ol} .2015 .3080$

\begin{abstract}
MicroRNAs are proposed to serve vital functions in the regulation of tumor progression and invasion. However, the expression levels of miR-203 in non-small cell lung cancer (NSCLC) and its clinical significance remain unknown. In the present study, the association between B-cell-specific moloney murine leukemia virus insertion site 1 (Bmil) and miR-203 was investigated. miR-203 was demonstrated to act as a tumor suppressor by regulating the expression of Bmil. miR-203 expression levels were downregulated in NSCLC tissues while Bmi1 expression was upregulated in NSCLC tissues and cell lines. Furthermore, downregulated Bmil or enhanced miR-203 expression inhibited NSCLC cell proliferation and invasion in vitro. In addition, a dual-luciferase reporter assay was performed, which identified Bmil as a novel target of miR-203. In conclusion, the present study demonstrated that miR-203 functions as a tumor suppressor and is important in inhibiting the proliferation of NSCLC cells through targeting Bmil. These findings indicate that miR-203 may be useful as a novel potential therapeutic target for NSCLC.
\end{abstract}

\section{Introduction}

Lung cancer is currently the most common type of cancer worldwide in terms of incidence and mortality, and non-small cell lung cancer (NSCLC) accounts for $85 \%$ of all lung cancer cases (1). There remains a lack of effective biomarkers or

Correspondence to: Dr Jun Zhao or Dr Chang Li, Department of Thoracic and Cardiovascular Surgery, The First Affiliated Hospital of Soochow University, Medical College of Soochow University, 188 Shizi Street, Suzhou, Jiangsu 215006, P.R. China

E-mail: zhaojia0327@126.com

E-mail: cli@suda.edu.cn

*Contributed equally

Key words: non small cell lung cancer, miR-203, Bmil, cell proliferation, invasion indicators for diagnosis, which often leads to delayed diagnosis and thus the majority of patients with NSCLC present with an advanced stage. Therefore, the development of novel techniques for early diagnosis of NSCLC is required.

MicroRNAs (miRNAs) are a class of small noncoding RNAs that are $19-24$ nucleotides in length, and they regulate $\sim 20-30 \%$ of the genes in the human genome (2). The coding sequences of miRNAs are located in the regions between genes or in the introns (3). Previous studies have demonstrated that miRNAs are involved in a variety of biological processes, including cell proliferation, differentiation, apoptosis and the development and differentiation of tissues and organs (4,5); and pathological processes, including tumorigenesis and tumor progression (6). Such regulation is achieved primarily by their partial recombination with the 3 -untranslated region (3'-UTR) of the target mRNA $(7,8)$.

A previous study revealed that molecular markers of cancer stem-like cells were connected with malignancies by using gene microarray and sequencing analysis (9). Another study demonstrated that miRNAs are critical in the carcinogenesis of lung cancer (10). miR-203 was first identified as a keratinocyte-specific miRNA in the skin, but it is also expressed in the squamous epithelium of the cervix and esophagus (11). miR-203 regulates embryonic epidermal differentiation and has been implicated in skin diseases, but it also serves as a tumor suppressor or oncogene by regulating proliferation, differentiation, invasion, metastasis and apoptosis in certain types of human cancer $(12,13)$. B-cell-specific moloney murine leukemia virus insertion site 1 (Bmil) is a member of the polycomb repressive complex 1 (PRC1) and is highly expressed in several types of cancer, including lung neoplasm $(14,15)$. Yu et al (9) demonstrated that miR-203 inhibited the proliferation of esophageal cancer cells by suppressing Bmil directly. However, the level of miR-203 expression and its role in NSCLC remains unclear.

In the present study, the interaction between miR-203 and Bmil expression levels was investigated, in addition to the mechanistic role of miR-203 in NSCLC.

\section{Materials and methods}

Patient sample collection. A total of 21 paired NSCLC samples were obtained from the First Affiliated Hospital of 
Table I. Primers for RT or amplification of the mature miR-203, U6, Bmi1 and GAPDH mRNA.

\begin{tabular}{ll}
\hline Name & \multicolumn{1}{c}{ Sequence, 5'-3' $^{\prime}$} \\
\hline RT primers & \\
U6 & CGAGCACAGAATCGCTTCACGAATTTGCGTGTCAT \\
miR-2031 & GTCGTATCCAGTGCAGGGTCCGAGGTATTCGCACTGGATACGACCTAGTGGTC \\
RT-qPCR primers & \\
U6 & F, CGAGCACAGAATCGCTTCA; and R, CTCGCTTCGGCAGCACATAT \\
miR-203 & F, GTATCCAGTGCAGGGTCCGA; and R, CGACGGTGAAATGTTTAG \\
Bmil & F, GTGCTTTGTGGAGGGTACTTCAT; and R, TTGGACATCACAAATAGGACAATACTT \\
GAPDH & F, GAAGGTGAAGGTCGGAGTC; and R, GAAGATGGTGATGGGATTTC
\end{tabular}

RT-qPCR, reverse transcription-quantitative polymerase chain reaction; Bmi1, B-cell-specific moloney murine leukemia virus insertion site 1.

Soochow University (Suzhou, China) between January 2013 and April 2014. All patients provided written informed consent prior to tissue donation for research purposes. The present study was approved by the ethics committee of Soochow University. All tissues were frozen in liquid nitrogen immediately following the operation and stored at $-70^{\circ} \mathrm{C}$ until required.

Cell culture. The human NSCLC cell lines A549, H1299, $\mathrm{H} 226, \mathrm{H} 1650, \mathrm{H} 460$ and LTEP- $\alpha-2$ were obtained from the Cell bank of the Chinese Academy of Sciences (Shanghai, China). The human bronchial epithelial (HBE) cell line was obtained from Bogoo Biological Technology Co. Ltd. (Shanghai, China). Cells were cultured in RPMI-1640 medium containing 10\% fetal bovine serum (FBS; Gibco Life Technologies, Carlsbad, CA, USA). All the cells were cultured in humidified sterile conditions containing $5 \% \mathrm{CO}_{2}$ at $37^{\circ} \mathrm{C}$.

Construction of the luciferase reporter plasmids, transfection and dual-luciferase assay. The psiCHECK-2 dual luciferase vector (Promega Corporation, Madison, WI, USA) was used to construct the plasmid containing the 3'-untranslated region (3'-UTR) of Bmil. The fragments containing the predicted wild and mutant sites were directly synthesized (Genewiz, Inc., Suzhou, China) and then subcloned into the psiCHECK-2 vector following digestion with XhoI and NotI restriction enzymes (Thermo Fisher Scientific, Inc., Pittsburgh, PA, USA) to generate the Bmil-3'-UTR-wild and Bmil-3'-UTR-mutant vectors. Subsequently, LTEP- $\alpha-2$ cells $\left(1 \times 10^{5} /\right.$ well $)$ were seeded into a 24-well plate and co-transfected with $50 \mathrm{ng} /$ well Bmi1-3'-UTR-wild or Bmi1-3'-UTR-mutant vector and $50 \mathrm{nM} /$ well miR-203 mimics (5'-UUGUGCUUGAUC UAACCAUGU-3') or scrambled microRNA negative control (miR-NC; 5'-UUCUCCGAACGUGUCACGUTT-3'). Following culture for $48 \mathrm{~h}$, the LTEP- $\alpha-2$ cells were collected and the luciferase activities were measured by the Dual-Luciferase Reporter Assay kit (Promega Corporation) on a TD20/20 Luminometer (Turner Designs, Westport, MA, USA). Each experiment was performed in triplicate. The results were expressed as relative Renilla luciferase activities, which were obtained following normalization to firefly luciferase activities. All the transient transfections were performed using Lipofectamine 2000 (Invitrogen Life Technologies, Carlsbad, CA, USA).
RNA extraction and reverse transcription-quantitative polymerase chain reaction $(R T-q P C R)$. The total RNA from cell lines and NSCLC tissues was extracted using TRIzol reagent (Invitrogen Life Technologies) and measured on a NanoDrop (ND-2000 spectrophotometer; Thermo Fisher Scientific, Inc., Wilmington, DE, USA ) according to the manufacturer's protocol. The synthesis of cDNA was performed using an M-MLV First Strand kit (Invitrogen Life Technologies). The RT primers for mature miR-203 and U6 were designed as RT stem-loop primers (Table I). Quantitative PCR (qPCR) was performed using a SYBR Green PCR kit (Takara Biotechnology Co., Ltd., Dalian, China) and an ABI7500 Real-Time system (Applied Biosystems Life Technologies, Foster City, CA, USA) were used to quantify the expression levels of RNA. U6 small nuclear RNA (snRNA) and GAPDH mRNA were used as endogenous controls to normalize miR-203 and Bmil expression levels. The primer sequences for miR-203, U6, Bmil and GAPDH detection are listed in Table I. The relative expression levels were calculated using the $\Delta \Delta \mathrm{Ct}$ method.

Western blot analysis. The cells were lysed in RIPA buffer (Cell Signaling Technology, Inc., Danvers, MA, USA) with protease inhibitor (Sigma-Aldrich, St. Louis, MO, USA) at $72 \mathrm{~h}$ post-transfection. The total proteins were separated by $10 \%$ SDS-PAGE (Sangon Biotech, Co., Ltd., Shanghai, China) and run at a constant voltage of $110 \mathrm{~V}$ for $2 \mathrm{~h}$, and subsequently transferred onto polyvinylidene difluoride membranes (Bio-Rad Laboratories, Inc., Hercules, California, USA). The membranes were blocked using $1 \%$ bovine serum albumin (Sigma-Aldrich) for $30 \mathrm{~min}$ and incubated with the primary antibody Bmil (monoclonal, rabbit anti-human; dilution, 1:3000; catalog no. 5856S; Cell Signaling Technology, Danvers, MA, USA) or GAPDH (polyclonal, rabbit anti-human; dilution, 1:5000; catalog no. AP0063; Bioworld Technology, Minneapolis, MN, USA) with agitation overnight at $4^{\circ} \mathrm{C}$. Following 3 washes with Tris-buffered saline and Tween-20 (TBST), the membranes were incubated with secondary antibodies (goat anti-rabbit, dilution, 1:3000; catalog no. sc-2004; Santa Cruz Biotechnology, Dallas, TX, USA) at room temperature for $2 \mathrm{~h}$ and then the washes were repeated. The result was visualized using an ECL detection system (Pierce Biotechnology, Inc., Rockford, IL, USA). The Bmil protein expression levels were normalized against the GAPDH protein expression levels 
A

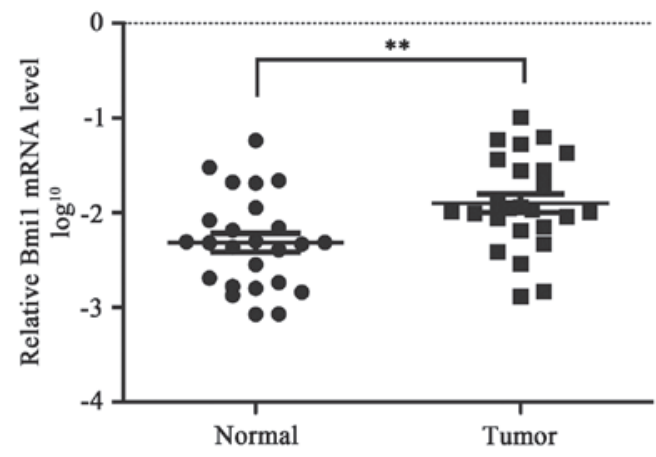

B

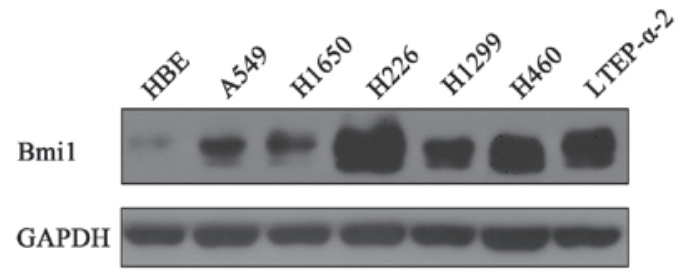

C

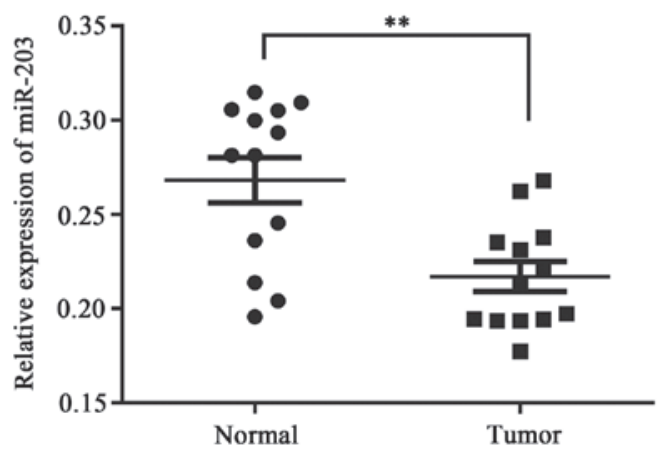

Figure 1. Bmil and miR-203 expression in NSCLC tissues and cells quantified by reverse transcription-quantitative polymerase chain reaction. (A) Bmil mRNA levels expressed in 25 NSCLC tissues and paired non-tumor lung tissues. (B) Western blot analysis of Bmil protein expression levels in HBE cells and NSCLC A549, H1650, H226, H1299, H460 and LTEP- $\alpha-2$ cells. GAPDH was used as internal control. (C) miR-203 levels expressed in 13 NSCLC tissues and paired non-tumor lung tissues. ${ }^{* *} \mathrm{P}<0.01$. Bmi1, B-cell-specific moloney murine leukemia virus insertion site 1; NSCLC, non-small cell lung cancer; HBE, human bronchial epithelial.

and the density was quantified using Quantity One software, version 4.6 (Bio-Rad Laboratories, Inc.).

Cell proliferation assay. When the cells were in the logarithmic growth phase, they were seeded into 96 -well plates at a density of $5 \times 10^{3}$ cells/well. Cell proliferation was detected using the Cell Counting kit-8 (CCK8) and 5-ethynyl-2'-deoxyuridine (EdU) assays. A CCK8 (Beyotime Institute of Biotechnology, Shanghai, China) was used following the manufacturer's instructions. To estimate the number of viable cells, the optical density at a wavelength of $450 \mathrm{~nm}$ (OD450) was measured daily over 4 consecutive days. The EdU assay (Guangzhou RiboBio, Co., Ltd., Guangzhou, China) was used to label cells undergoing DNA replication. At $48 \mathrm{~h}$ post-transfection, the cells were cultured with EdU reagent (Guangzhou RiboBio Co., Ltd.) for $2 \mathrm{~h}$ to identify those cells in the S-phase of the cell cycle. The EdU medium mixture was then discarded and the cells were fixed with $4 \%$ paraformaldehyde (Sigma-Aldrich) for $30 \mathrm{~min}$ at room temperature. Following washes with glycine $(2 \mathrm{mg} / \mathrm{ml}$; Sigma-Aldrich) and phosphate-buffered saline for $5 \mathrm{~min}$ each, $0.5 \%$ Triton X-100 (Sigma-Aldrich) was added to each well. Apollo dyeing reaction buffer (Guangzhou RiboBio, Co., Ltd.) was then added to each well and the plate was shaken in the dark for $30 \mathrm{~min}$ at room temperature. The DNA was stained by Hoechst 33342 (Guangzhou RiboBio, Co., Ltd.) dye for $30 \mathrm{~min}$ and the proportion of nucleated cells coalescent with EdU were observed using fluorescence microscopy (Olympus IX71; Olympus, Tokyo, Japan).

Apoptosis assay. The cells were collected following $48 \mathrm{~h}$ of culture post-transfection with miR-203 and the cell density was adjusted to $1 \times 10^{6} \mathrm{cells} / \mathrm{ml}$. The cells were then stained using an Annexin V-FITC Apoptosis Detection kit (Beyotime Institute of Biotechnology) according to the manufacturer's protocol. The level of apoptosis was assessed by flow cytometry (FC500; Beckman Coulter, Miami, FL, USA).

Transwell invasion assay. At $24 \mathrm{~h}$ post-transfection with miR-203 or miR-NC, the cells were harvested. For the invasion assay, the cells were resuspended in RPMI-1640 medium containing $1 \%$ FBS and then seeded at a density of $4 \times 10^{4}$ cells/well into the inserts of the Transwell chamber (Corning Incorporated, Corning, NY, USA), which were coated with Matrigel (27.2 $\mu \mathrm{g} / \mu \mathrm{l}$; BD Biosciences, Franklin Lakes, NJ, USA). The inserts were subsequently cultured in wells with $20 \%$ FBS-containing medium and the plates were incubated at $37^{\circ} \mathrm{C}$ for $36 \mathrm{~h}$. Next, the inserts were removed and a cotton tip was used to scrape off the non-invading cells on the upper surface. Cells on the lower surface were then fixed with $4 \%$ paraformaldehyde for $30 \mathrm{~min}$ at room temperature, and stained with $0.1 \%$ crystal violet (Sigma-Aldrich) for a further $30 \mathrm{~min}$. Following fixation, 3 randomly selected images per well were acquired using an inverted microscope (Olympus CKX41; Olympus, Tokyo, Japan) and the average counts were calculated.

Statistical analysis. The results were analyzed using GraphPad Prism Software, version 5.01 (GraphPad Software Inc., La Jolla, CA, USA). All data are presented as the mean \pm standard deviation from 3 independent experiments. A 2-tailed Student's t-test was used for the comparison of the mean between groups. $\mathrm{P}<0.05$ was considered to indicate a statistically significant difference.

\section{Results}

Upregulation of Bmil and downregulation of $\mathrm{miR}-203$ in NSCLC. To determine whether the expression of Bmil was increased in NSCLC, the Bmil mRNA expression in 25 paired NSCLC tissues and adjacent non-tumor tissues was quantified by RT-qPCR. This analysis revealed that the mRNA expression level of Bmil was significantly increased in NSCLC tissues compared with paired non-tumor tissues $(\mathrm{P}<0.01$; Fig. 1A). In addition, Bmil protein levels were measured in 7 different cell lines. Compared with HBE cells, Bmil protein expression levels were significantly increased in the NSCLC cell lines, including the A549, H1650, H226, H1299, H460 and 
A

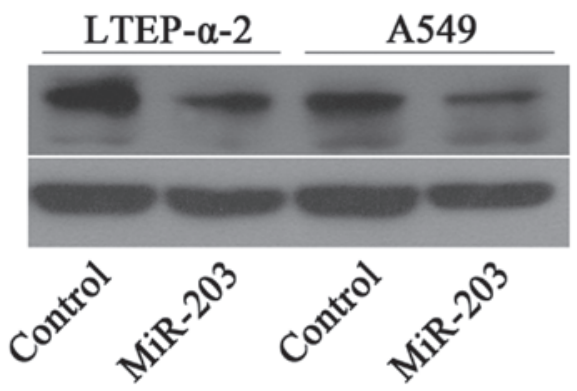

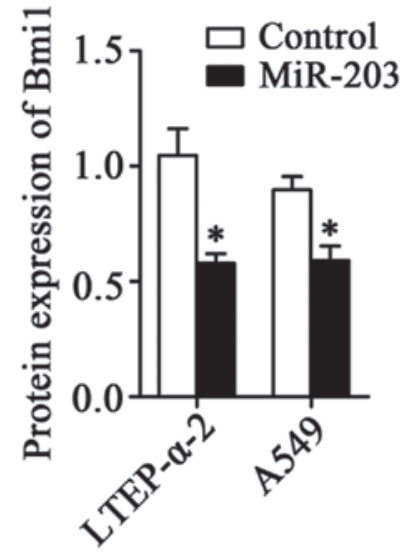

B

Hsa -AAAAUUGUAGCUAAACAUUUCAUUGU--CCCCAGUCUGCA--
Ptr -AAAAUUGUAGCUAAACAUUUCAUUGU--CCCCAGUCUGCA--
Mml -AAAAUUGUAGCUAAACAUUUCAUUGU--CCCCAGUCUGCA--
Oga -AAAAUUGUAGCUAAACAUUUCAUUGU--CCCCAGUCUGCA--
Mmu --AAACUGUAGCUAAGCAUUUCAUUGU--CUGCAGUCUGCA--
Rno --ACCCUGUAGCUAAGCAUUUCAUGGU--CUACAGUCUGCA--

C
MiR-203
3'-GAUCACCAGGAUUUGUAAAGUG-5'
Bmil 3' UTR (1443-1449)
5'...AAAAUUGUAGCUAAACAUUUCAU....3'
Bmil 3' UTR-mut
$5^{\prime} \ldots$ AAAAUUGUAGCUAAACTUCUG ${ }_{*}{ }_{*}$

D

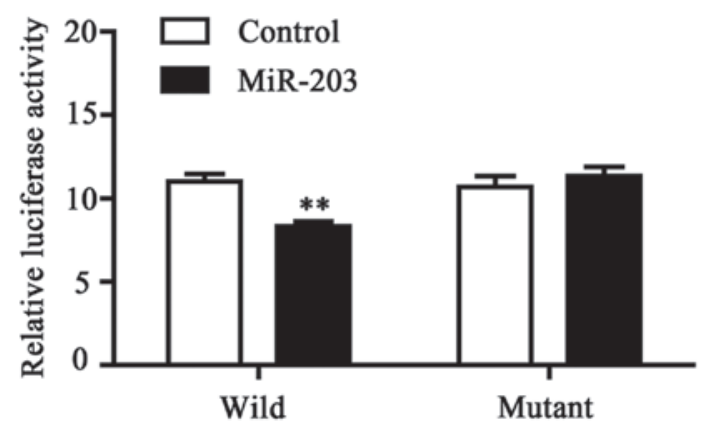

Figure 2. Bmil is a direct target of miR-203. (A) Western Blot analysis was performed to detect the expression of Bmi1 in NSCLC LTEP- $\alpha-2$ and A549 cells transfected with miR-203 mimics or miR-NC (control). (B) Sequence alignment of predicted miR-203 target sites on Bmil 3'-UTR displaying high conservation between human and other species. (C) Potential association between the predicted miR-203 target sites and miR-203. The asterisks indicate the replaced bases in Bmi1-3'-UTR-mutant vectors. (D) LTEP- $\alpha$-2 cells were co-transfected with miR-203 mimics or miR-NC and psiCHECK-2 vector with Bmi1 3'-UTR-wild or mutant. Renilla luciferase activity was obtained following normalization to firefly luciferase activity. ${ }^{*} \mathrm{P}<0.05$; ${ }^{* *} \mathrm{P}<0.01$ vs. control. Bmi1, B-cell-specific moloney murine leukemia virus insertion site 1; NSCLC, non-small cell lung cancer; UTR, untranslated control.

LTEP- $\alpha-2$ cell lines (Fig. 1B). However, miR-203 was significantly downregulated in the 13 NSCLC samples compared with paired non-tumor tissues (Fig. 1C).

Bmil is a functional target of miR-203 in NSCLC. Bmil is a functional target of miR-203 in NSCLC. Using TargetScan software, the potential targets of miR-203 including SNAI2, PKC $\alpha$, Bmi1, PLD2, LASP1 and c-myc were identified. To evaluate whether miR-203 regulates Bmil expression, miR-203 mimics were transiently transfected into A549 and LTEP- $\alpha-2$ cells. The results demonstrated that the overexpression of miR-203 markedly reduced Bmil protein expression levels in LTEP- $\alpha-2$ and A549 cells $(\mathrm{P}<0.05$; Fig. $2 \mathrm{~A})$. In accordance with these findings, it is possible that miR-203 directly targets Bmil to inhibit its expression. To investigate this hypothesis, potential targets of miR-203 were identified using TargetScan software (www.targetscan.org) and a highly conserved predicted target site on the 3'UTR of Bmil mRNA was identified (Fig. 2B). This region of the Bmil 3'UTR, including the predicted miR-203 binding site, was then cloned into the psiCHECK-2 vector (Fig. 2C). The results of the dual-luciferase assay demonstrated that in LTEP- $\alpha-2$ cells co-transfected with the Bmil-3'-UTR-wild vector, miR-203 significantly suppressed the luciferase activity compared with the control group. However, this result was not observed with the Bmi1-3'-UTR-mutant vector. These results indicate 


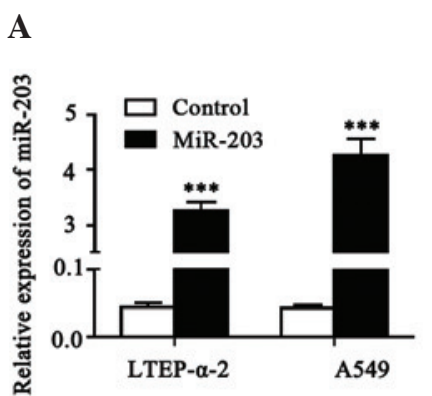

D
$\mathbf{B}$

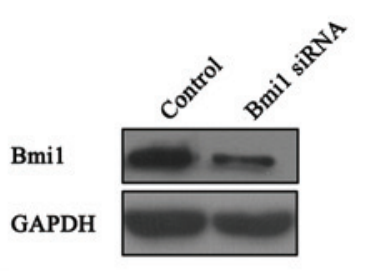

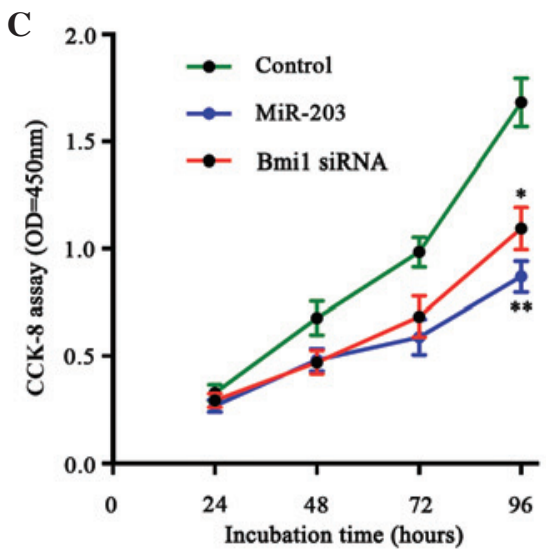

E

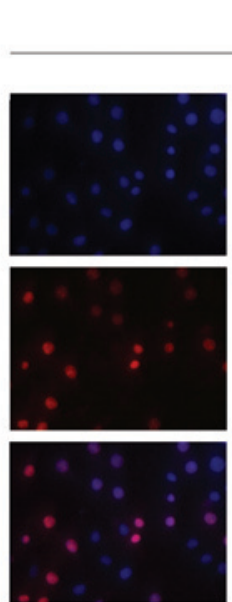

Control
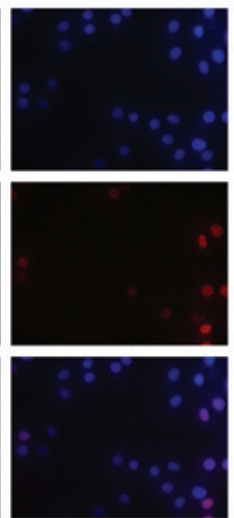

MiR-203
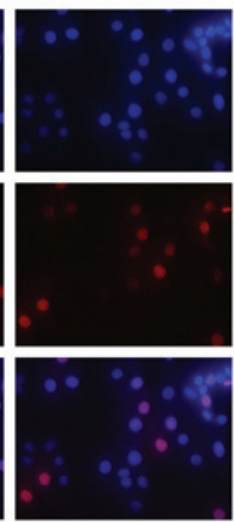

Bmil siRNA
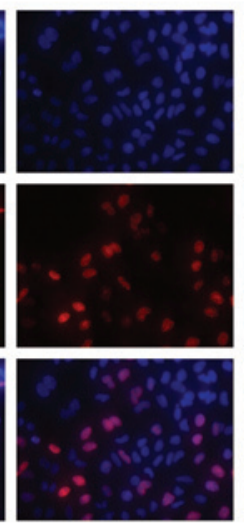

Control
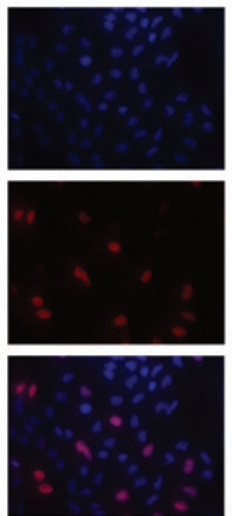

MiR-203
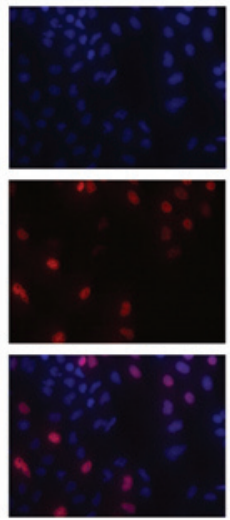

Bmil siRNA
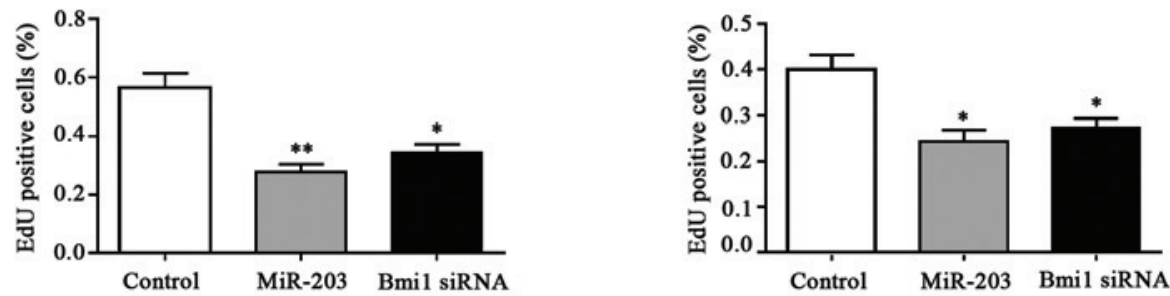

Figure 3. Effects of transfection of miR-203 and Bmil siRNA on the proliferation of NSCLC cells. (A) At 48 h post-transfection with miR-203 mimics or miR-NC (control) in LTEP- $\alpha-2$ and A549 cells, miR-203 expression was quantified by qPCR analysis. (B) Western blot analysis demonstrated that Bmil protein expression was knocked down by Bmil-siRNA transfection. (C) CCK8 analysis demonstrated that cell proliferation in LTEP- $\alpha-2$ cells was inhibited by transfection with miR-203 or Bmil-siRNA. At $48 \mathrm{~h}$ post-transfection with miR-203 mimics or Bmil-siRNA EdU assay was applied in (D) LTEP- $\alpha-2$ and (E) A549 cells. The percentage of EdU-positive cells in each group was calculated and $\geq 200$ cells were counted per well. ${ }^{*} \mathrm{P}<0.05$; ${ }^{* * *} \mathrm{P}<0.01{ }^{* * * *} \mathrm{P}<0.001 \mathrm{vs}$. control. Bmil, B-cell-specific moloney murine leukemia virus insertion site; NSCLC, non-small cell lung cancer; qPCR, quantitative polymerase chain reaction; EdU, 5-ethynyl-2'-deoxyuridine.

that miR-203 binds to the 3'-UTR of Bmil mRNA and thus regulates its expression (Fig. 2D).

miR-203 inhibits cell proliferation in NSCLC cells. The effects of miR-203 expression on malignant phenotypes of NSCLC cells were observed. The efficiency of transfection was determined by RT-qPCR, demonstrating increased expression levels of miR-203 in the mimics-transfected cells compared with the control cells $(\mathrm{P}<0.01$; Fig. $3 \mathrm{~A})$. In addition, the protein level of Bmil was successfully suppressed by Bmi1-siRNA, as demonstrated by western blot analysis (Fig. 3B). The results of the CCK8 assays demonstrated that ectopic miR-203 expression significantly inhibited LTEP- $\alpha-2$ cell growth compared with the control group $(\mathrm{P}<0.01$; Fig. 3C). A similar result was observed following the suppression of Bmil expression using Bmil-siRNA $(\mathrm{P}<0.05$; Fig. 3C). The EdU incorporation assay also indicated that miR-203 inhibited NSCLC cell proliferation. Ectopic expression of miR-203 significantly reduced EdU incorporation in LTEP- $\alpha-2$ (P<0.01; Fig. 3D) and A549 (P<0.05; Fig. 3E) cells, 
A
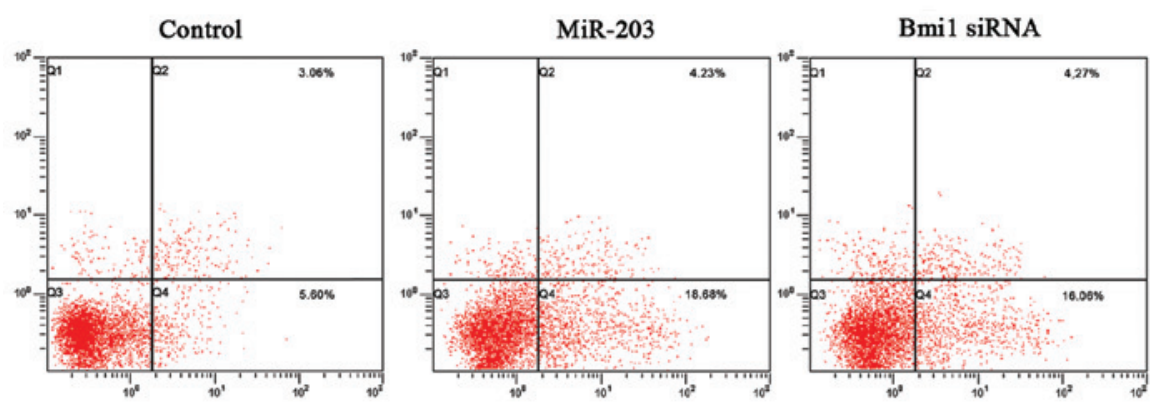

C
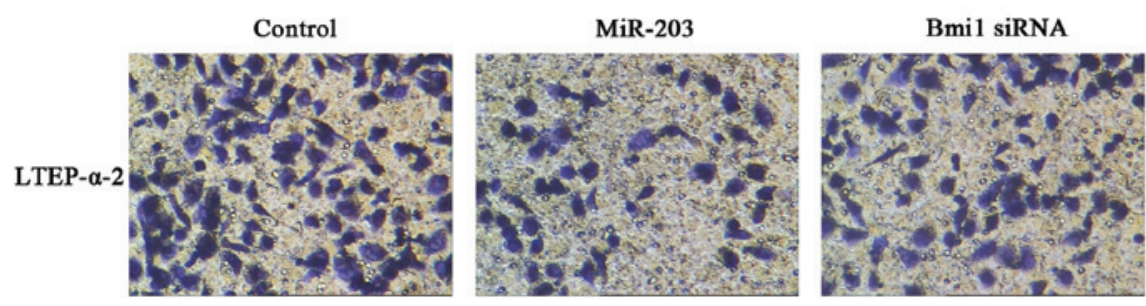
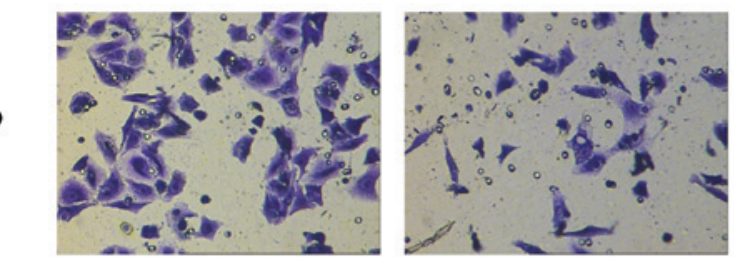

A549

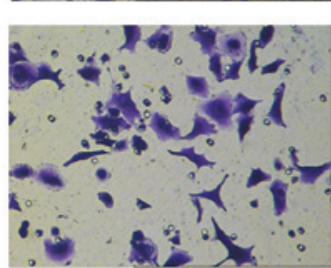

B

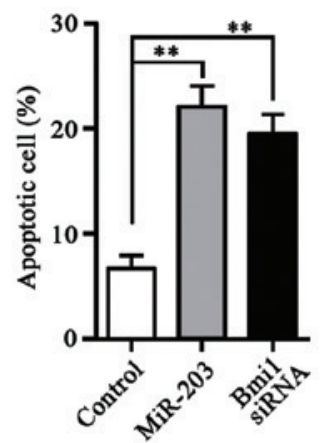

D
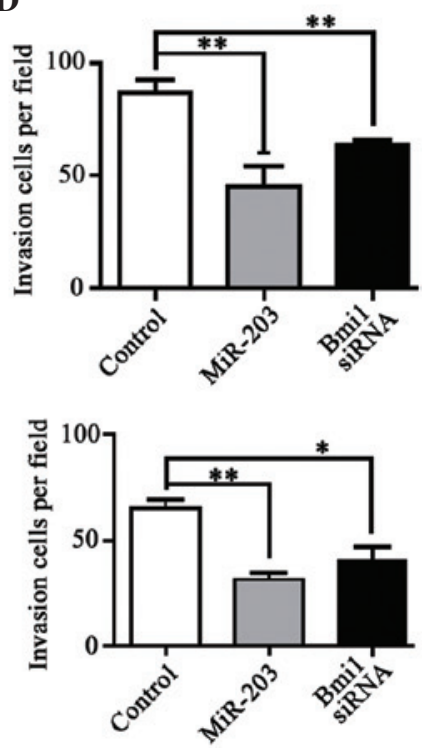

Figure 4. (A) Flow cytometric analysis of LTEP- $\alpha-2$ cells following transfection with miR-203 or Bmil siRNA. Q2 + Q4 represents the total apoptosis rate. (B) Percentage of apoptotic cells. Each experiment was performed at least in triplicate. Following transient transfection with miR-203 or Bmil-siRNA, Transwell invasion assay was performed in LTEP- $\alpha-2$ and A549 cells. (C) Crystal violet staining and (D) quantification of cell invasion. "P $<0.05$; ${ }^{* *} \mathrm{P}<0.01$. Bmil; B-cell-specific moloney murine leukemia virus insertion site 1.

which is in accordance with the reduction in EdU incorporation induced by attenuated expression of Bmil with specific siRNA in LTEP- $\alpha-2(\mathrm{P}<0.05$; Fig. 3D) and A549 $(\mathrm{P}<0.05$; Fig. 3E) cells. These results indicate that forced expression of miR-203 resulted in significant inhibition of cell growth in NSCLC cells in vitro.

Restoration of miR-203 induces apoptosis in NSCLC cells. An apoptosis assay was performed in LTEP- $\alpha-2$ cells with increased miR-203 expression or silenced Bmil (Fig. 4A). The percentage of apoptotic cells in the miR-203 transfection group was significantly increased compared with the control group (21.7 vs. $8.7 \%, \mathrm{P}<0.01$; Fig. 4B). Similar to the results obtained from the miR-203 transfection, comparable results were obtained from downregulation of Bmil, in which the percentage of apoptotic cells was also significantly increased compared with the control group (20.3 vs. $8.7 \%, \mathrm{P}<0.01$; Fig. 4B). The flow cytometry results demonstrated that ectopic expression of miR-203 and downregulation of Bmil by siRNA induced cell apoptosis.

Upregulation of miR-203 inhibited metastatic ability of NSCLC cells. To demonstrate whether miR-203 expression affects the metastatic capability of NSCLC cells, a Transwell invasion assay was performed (Fig. 4C). The quantity of LTEP- $\alpha-2$ and A549 cells that invaded the filter in the miR-203 transfected group was markedly reduced compared with the control group in the two cell types $(\mathrm{P}<0.01 ;$ Fig. 4D). Similarly, cell invasion was significantly reduced following transfection of Bmil-specific siRNA ( $\mathrm{P}<0.01$ and $\mathrm{P}<0.05$ in LTEP- $\alpha-2$ and A549 cells, respectively; Fig. 4D).

\section{Discussion}

MicroRNAs are a class of small noncoding RNAs that serve important functions in the regulation of the biological processes of cells, including differentiation, proliferation and apoptosis (16). Previous studies have demonstrated that miRNA dysregulation occurs in various types of human cancer and these aberrantly expressed miRNAs are considered to serve pivotal roles in carcinogenesis (17-19). Whether miRNAs act as oncogenes or tumor suppressor genes depends on the context. For example, miR-203 behaves as a tumor suppressor and is downregulated in pancreatic, glioma and esophageal cancer, while it is upregulated in epithelial ovarian cancer and acts as an oncogene (9,20-22).

The present study demonstrated that miR-203 was downregulated in NSCLC specimens, which is in agreement with a 
previous study (23). The inhibitory effect of miR-203 expression on the proliferation and invasion of NSCLC cells that was observed in the present study indicates that miR-203 may be involved in the tumorigenesis of lung cancer. Future studies should aim to investigate whether miR-203 expression affects cell cycle progression and can induce cell apoptosis in NSCLC.

A previous study demonstrated that downregulated miR-203 levels were significantly associated with lymph node metastasis in laryngeal squamous cell carcinoma (24). However, to the best of our knowledge, no studies have yet compared the association between miR-203 and lymph node metastasis in lung cancer. The ability of tumor cells to migrate and invade is associated with their cell adhesion properties $(25,26)$. The results of the present study indicate that the restoration of miR-203 expression may serve an important role in the progress of NSCLC treatment through inhibiting cell invasion, though further in vivo studies are required to confirm this.

To understand the mechanisms by which miR-203 suppresses cell proliferation, TargetScan software was used to predict the target genes of miR-203. SNAI2, PKC $\alpha$, Bmi1, PLD2, LASP1 and $c$-myc were identified in the present study as potential targets. A proportion of these targets have already been demonstrated to be directly regulated by miRNAs, for example SNAI2 expression is reported to be repressed by miR-203 in prostate cells (27). SNAI2 is a transcription factor that belongs to the E-box-motif family. It inhibits apoptosis by repressing $\mathrm{p} 53$-mediated transcription and promotes epithelial-mesenchymal transition by directly repressing E-cadherin $(28,29)$. However, it is not clear whether there is an interaction between miR-203 and Bmil in NSCLC. Bmil, a polycomb gene family member, is expressed in almost all tissues and is upregulated in various types of human cancer indicating that Bmil may be involved in tumor progression (14). A previous study demonstrated that Bmil is a direct target of miR-128 in glioma and that it serves an important function in glioma proliferation and self-renewal (30). Another study demonstrated that Bmi1 was overexpressed in lung cancer (15), which is in accordance with the results of the present study. The present study also demonstrated Bmil protein expression levels were significantly increased in the NSCLC cell lines, A549, H1299, H226, H1650, H460 and LTEP- $\alpha-2$, compared with healthy HBE cells.

To assess whether miR-203 directly represses Bmil expression, western blot analysis and a luciferase assay were performed. These analyses corroborated that miR-203 specifically downregulated endogenous Bmil expression at the post-transcriptional level.

There are numerous genes predicted to be targets of miR-203, and Bmil was the only one confirmed by the present study. There may be other potential targets of miR-203 that function in the tumorigenesis of lung cancer, such as $c$-myc, therefore further studies focusing on these novel target genes are required. In addition, the sample size in the present study was small, and further studies from different specimens of NSCLC patients are required to strengthen the findings of the present study.

In conclusion, the present study demonstrated that miR-203 represses Bmil protein expression by directly targeting the Bmi1 3'UTR, which may reduce the proliferative and invasive abilities of NSCLC cells. These findings indicate that miR-203 may be a novel target for the diagnosis and therapy of NSCLC.

\section{Acknowledgements}

The authors would like to thank the patients for their participation and cooperation and Dr Hong-Tao Zhang for his helpful criticism. The present study was supported in part by the grants from the Jiangsu Province Key Provincial Talents Program (grant no. RC2011106 to Dr Jun Zhao), the Provincial Talents of Six Summits Program (the eighth, grant no. 2011-WS-056 to Dr Jun Zhao), the Natural Science Foundation of Jiangsu Province (grant no. BK20131159 to Dr Jun Zhao), Suzhou city Municipal Youth Fund of Science and Education (grant no. KJXW2011007 to Dr Chang Li) and the Suzhou Key Laboratory for Molecular Cancer Genetics (grant no. SZS201209 to Dr Jun Zhao).

\section{References}

1. Li C, Xu C, Ma H, et al: Video-assisted thoracoscopic lobectomy with a single utility port is feasible in the treatment of elderly patients with peripheral lung cancer. Thorac Cancer 5: 219-224, 2014.

2. Shi Y, Liu C, Liu X, et al: The microRNA miR-34a inhibits non-small cell lung cancer (NSCLC) growth and the CD44hi stem-like NSCLC cells. PLoS One 9: e90022, 2014.

3. Monteys AM, Spengler RM, Wan J, et al: Structure and activity of putative intronic miRNA promoters. RNA 16: 495-505, 2010.

4. Kasinski AL and Slack FJ: Epigenetics and genetics. MicroRNAs en route to the clinic: progress in validating and targeting microRNAs for cancer therapy. Nat Rev Cancer 11: 849-864, 2011.

5. Croce CM and Calin GA: miRNAs, cancer, and stem cell division. Cell 122: 6-7, 2005.

6. Fang YX and Gao WQ: Roles of microRNAs during prostatic tumorigenesis and tumor progression. Oncogene 33: 135-147, 2014.

7. Lagos-Quintana M, Rauhut R, et al: Identification of novel genes coding for small expressed RNAs. Science 294: 853-858, 2001.

8. Lee RC and Ambros V: An extensive class of small RNAs in Caenorhabditis elegans. Science 294: 862-864, 2001.

9. Yu X, Jiang X, Li H, et al: miR-203 inhibits the proliferation and self-renewal of esophageal cancer stem-like cells by suppressing stem renewal factor Bmi-1. Stem Cells Dev 23: 576-585, 2014.

10. Esquela-Kerscher A and Slack FJ: Oncomirs - microRNAs with a role in cancer. Nat Rev Cancer 6: 259-269, 2006.

11. Sonkoly E, Wei T, Janson PC, et al: MicroRNAs: novel regulators involved in the pathogenesis of psoriasis? PLoS One 2: e610, 2007.

12. Yu J, Li A, Hong SM, Hruban RH and Goggins M: MicroRNA alterations of pancreatic intraepithelial neoplasias. Clin Cancer Res 18: 981-992, 2012.

13. Jin J, Deng J, Wang F, et al: The expression and function of microRNA-203 in lung cancer. Tumour Biol 34: 349-357, 2013.

14. Jiang L, Li J and Song L: Bmi-1, stem cells and cancer. Acta Biochim Biophys Sin (Shanghai) 41: 527-534, 2009.

15. Meng X, Wang Y, Zheng X, et al: shRNA-mediated knockdown of Bmi-1 inhibit lung adenocarcinoma cell migration and metastasis. Lung Cancer 77: 24-30, 2012.

16. Wang Z, Yao H, Lin S, et al: Transcriptional and epigenetic regulation of human microRNAs. Cancer Lett 331: 1-10, 2013.

17. He L, Thomson JM, Hemann MT, et al: A microRNA polycistron as a potential human oncogene. Nature 435: 828-833, 2005.

18. Bartel DP: MicroRNAs: genomics, biogenesis, mechanism, and function. Cell 116: 281-297, 2004.

19. Iorio MV, Visone R, Di Leva G, et al: MicroRNA signatures in human ovarian cancer. Cancer Res 67: 8699-8707, 2007.

20. Miao L, Xiong X, Lin Y, et al: miR-203 inhibits tumor cell migration and invasion via caveolin-1 in pancreatic cancer cells. Oncol Lett 7: 658-662, 2014.

21. Dontula R, Dinasarapu A, Chetty C, et al: MicroRNA 203 modulates glioma cell migration via Robo1/ERK/MMP-9 signaling. Genes Cancer 4: 285-296, 2013.

22. Wang S, Zhao X, Wang J, et al: Upregulation of microRNA-203 is associated with advanced tumor progression and poor prognosis in epithelial ovarian cancer. Med Oncol 30: 681, 2013. 
23. Wang C, Wang X, Liang H, et al: miR-203 inhibits cell proliferation and migration of lung cancer cells by targeting PKC $\alpha$ PLoS One 8: e73985, 2013.

24. Tian L, Li M, Ge J, et al: MiR-203 is downregulated in laryngeal squamous cell carcinoma and can suppress proliferation and induce apoptosis of tumours. Tumour Biol 35: 5953-5963, 2014.

25. Torres VA: Rab'ing tumor cell migration and invasion: Focal adhesion disassembly driven by Rab5. Cell Adhes Migr 8: 84-87, 2014.

26. Li L, Zhao F, Lu J, et al: Notch-1 signaling promotes the malignant features of human breast cancer through NF- $\kappa$ B activation. PLoS One 9: e95912, 2014

27. Qu Y, Li WC, Hellem MR, et al: MiR-182 and miR-203 induce mesenchymal to epithelial transition and self-sufficiency of growth signals via repressing SNAI2 in prostate cells. Int J Cancer 133: 544-555, 2013.
28. Pérez-Caro M, Bermejo-Rodríguez C, González-Herrero I, et al: Transcriptomal profiling of the cellular response to DNA damage mediated by Slug (Snai2). Br J Cancer 98: 480-488, 2008.

29. Peinado H, Olmeda D and Cano A: Snail, Zeb and bHLH factors in tumour progression: an alliance against the epithelial phenotype? Nat Rev Cancer 7: 415-428, 2007.

30. Godlewski J, Nowicki MO, Bronisz A, et al: Targeting of the Bmi-1 oncogene/stem cell renewal factor by microRNA-128 inhibits glioma proliferation and self-renewal. Cancer Res 68: 9125-9130, 2008. 